\title{
Multimodality MR Imaging Findings of Low-Grade Brain Edema in Hepatic Encephalopathy
}

\author{
L.J. Zhang, J. Zhong, and G.M. Lu
}

\begin{abstract}
SUMMARY: HE is a neuropsychiatric syndrome that develops in patients with severe liver diseases, with portosystemic shunt surgery in the form of diffuse mild brain edema. It is also associated with functional changes, such as those in attention and the DMN. MR imaging offers a range of capabilities for assessing the low-grade brain edema and its functional changes in brain affected by HE; therefore, it provides the opportunity to uncover the pathophysiologic mechanisms of HE. This article will review our current understanding of the pathophysiology of low-grade brain edema and will outline the role of structural MR imaging, MTR, DWI, DTI, and MR spectroscopy in the detection of low-grade brain edema, and the role of BOLD FMRI in the exploration of the related functional changes. A perspective of the study in this area will also be provided.
\end{abstract}

ABBREVIATIONS: ALFF = amplitude of low-frequency fluctuation; BOLD = blood oxygen level-dependent; DMN $=$ default mode network; FA $=$ fractional anisotropy; $\mathrm{FDR}$ = false discovery rate; Glx = glutamine/glutamate; ${ }^{\mathrm{H}} \mathrm{H}-\mathrm{MR}=$ proton MR; IPL = inferior parietal cortex; $\mathrm{MHE}=$ minimal hepatic encephalopathy; $\mathrm{mlns}=$ myo-inositol; $\mathrm{MTI}=$ magnetic transfer imaging; $\mathrm{MTR}=$ magnetic transfer ratio; $\mathrm{NAA}=\mathrm{N}$-acetylaspartate; $\mathrm{NCT}=$ number connection test; $\mathrm{PCC}=$ posterior cingulate cortex

$\mathbf{H}^{\mathrm{s}}$

$\mathrm{E}$ is a neuropsychiatric syndrome that develops in patients with severe liver diseases and/or portosystemic shunt surgery resulting from a serious complication of acute and chronic liver failure. HE is characterized by a wide spectrum of clinical manifestations, ranging from alterations of psychometric performance to stupor and coma. ${ }^{1} \mathrm{HE}$ is regarded as the result of overt brain edema and increased intracranial pressure in acute liver failure. However, patients with cirrhosis and HE usually show no clinical signs of brain edema and increased intracranial pressure, instead in vivo and in vitro studies from humans and animals have demonstrated the existence of a low-grade brain edema. ${ }^{2-10}$ This review will focus first on the roles of low-grade brain edema in $\mathrm{HE}$ and its qualitative and quantitative diagnosis with advanced multimodal MR imaging techniques. We will then review the results

From the Department of Medical Imaging (L.J.Z., G.M.L.), Jinling Hospital, Clinical School of Medical College, Nanjing University, Nanging, Jiangsu Province, China; and Department of Imaging Sciences (J.Z.), University of Rochester School of Medicine and Dentistry, Rochester, New York.

This work was supported by grants received from the Natural Scientific Foundation of China (30700194 and 81171313 to L.J.Z.) and the Natural Scientific Foundation of Jiangsu Province of China (BK2007572 to L.J.Z.).

Please address correspondence to Guang Ming Lu, MD, Department of Medical Imaging, Jinling Hospital, Clinical School of Medical College, Nanjing University, 305 Zhongshan East Rd, Xuanwu District, Nanjing, Jiangsu Province, China, 210002; email: cjr.luguangming@vip.163.com

- Indicates open access to non-subscribers at www.ajnr.org

http://dx.doi.org/10.3174/ajnr.A2968 of functional changes that are attributed to brain edema in patients with cirrhosis and HE by using BOLD fMRI.

\section{CLINICAL FEATURES OF HE}

The Working Party at the 11th World Congresses of Gastroenterology held in Vienna in 1998 recommended the nomenclature and types of HE: HE type A, encephalopathy associated with acute liver failure; HE type B, encephalopathy associated with portosystemic bypass and no intrinsic hepatocellular disease; and HE type C, encephalopathy associated with cirrhosis and portal cirrhosis and portal hypertension or portosystemic shunts. ${ }^{11} \mathrm{HE}$ type $\mathrm{C}$ can be classified into 3 main groups on the basis of the duration and characteristics of the clinical manifestations: episodic, chronic, and minimal. Episodic HE is characterized by the development of a confusional syndrome, including impaired mental state, neuromuscular abnormalities, asterixis, fetor hepaticus, and hyperventilation, which develops during a short period and fluctuates in severity. Chronic HE can be further classified into subgroups: relapsing $\mathrm{HE}$ and persistent HE. Relapsing HE manifests as frequent episodes of acute HE, while persistent HE refers to manifestations that do not reverse despite adequate treatment. The most characteristic manifestations of severe persistent HE are dementia, parkinsonism, or myelopathy in combination with other manifestations of neurologic involvement (ataxia, gait abnormalities, tremor). ${ }^{1}$

Recently, MHE becomes the focus of study in the HE field because it causes impairment of driving and daily living 
and occurs in $20 \%-80 \%$ of patients with cirrhosis (especially in patients with advanced liver disease, Child-Pugh B/C). ${ }^{3} \mathrm{MHE}$ refers to the population of patients with cirrhosis or portosystemic shunts who have subtly abnormal cognitive and/or neurophysiologic functions but no significant clinical signs and symptoms of HE. MHE was also diagnosed in those patients with extrahepatic portal venous obstruction. ${ }^{12}$ The neuropsychological features of MHE point to a disorder of executive functioning, particularly selective attention and psychomotor speed; but other abnormalities, such as working memory, are also seen. ${ }^{13,14} \mathrm{~A}$ complete psychometric assessment by a neuropsychologist is the best way to know the extent of the patient's cognitive impairment. A standardized test battery, including the NCT-A and NCT-B, line-tracing, serial-dotting, and digit-symbol tests (portosystemic encephalopathy syndrome test), has a high specificity for $\mathrm{HE}$ compared with other metabolic encephalopathies. ${ }^{11}$ Some authors believe that critical flicker frequency is a simple, reliable, and accurate test without any age or literacy dependence for the diagnosis of MHE and could predict the development of overt HE. ${ }^{15}$ However, changes in electroencephalogram/evoked responses and neuroimaging findings are nonspecific and may not be able to provide sufficient information for the diagnosis of minimal HE.

\section{LOW-GRADE BRAIN EDEMA AND HE}

Clinically manifest brain edema is a well-known complication of acute or fulminant hepatic failure; however, patients with cirrhosis and HE usually show no clinical signs of overt brain edema. In the 1990s, in vivo ${ }^{1} \mathrm{H}-\mathrm{MR}$ spectroscopy studies provided the first evidence for the presence of low-grade brain edema in these patients with cirrhosis and $\mathrm{HE}$ in the form of depletion of the mIns peak and elevation of the Glx signal intensity. ${ }^{16}$ Since then, many studies have shown the presence of low-grade brain edema as a result of astrocyte swelling and the role of ammonia and HE precipitating factors, such as neuroinflammation processes in the pathogenesis of HE. ${ }^{9,17}$

Various hypotheses have been proposed to explain the complex neuropsychiatric syndrome seen in HE. Hyperammonemia is the most important and widely accepted hypothesis for HE. ${ }^{1,5,6,8}$ The hyperammonemia leads to profound astrocyte changes, including Alzheimer type II changes in chronic HE and astrocyte swelling in acute HE. There is convincing evidence from animal and imaging studies for the role of brain edema as a major contributor to the development of HE. ${ }^{3-7}$ Brain edema is partly due to uptake of ammonia into astrocytes causing cellular swelling as 1 common pathogenetic end point. In addition, oxidative stress, endogenous benzodiazepine-like ligands, inflammatory cytokines, and potential manganese toxicity can all have a role in the pathogenesis of HE. However, no single factor can fully explain this pathogenesis. More recent evidence suggests that there is a synergistic effect between ammonia and various other inflammatory cytokines that results in excess glutamine within astrocytes, leading to osmotic swelling of the astrocytes and the subsequent brain edema as well as other neurocytotoxic effects. ${ }^{8-10}$ Haüssinger and Schliess ${ }^{18}$ proposed a model of the pathogenesis of HE. In this model, ammonia and other neurotoxins synergistically induce a low-grade cerebral edema as a result of astrocyte swelling, which is sufficient to trigger multiple alterations of astrocyte function and gene expression. As a result of an altered astrocyte function, glial neuronal communication and multiple neurotransmitter systems become deranged and impact synaptic plasticity and cerebral networks, which finally may account for the symptoms of HE (Fig 1). ${ }^{18}$

\section{QUALITATIVE AND QUANTITATIVE MR IMAGING OF BRAIN EDEMA IN HE \\ Brain Edema in Acute HE}

Patients with acute HE may exhibit characteristic regions of involvement on T2-weighted and FLAIR sequences. ${ }^{19-21}$ These patients having diffuse cortical involvement from imaging findings are more likely to have a poor outcome, though even such diffuse insults may ultimately reverse. ${ }^{20,21} \mathrm{U}$-King-Im et $\mathrm{al}^{21}$ reported a specific pattern of imaging findings, with extensive cortical injury, including bilateral symmetric involvement of the insular and cingulate cortices. Additional involvement of parietal, frontal, temporal, or occipital cortices was seen but was much more variable in extent and also asymmetric. In acute HE, diffuse cortical restricted diffusion resulting from cytotoxic edema can be detected, with the appearance of a decreased ADC value on the ADC map and high signal intensity on DWI in diffuse regions of the cerebral cortex. ${ }^{19-21}$ The MR imaging extent on FLAIR and DWI strongly correlate with the severity and duration of the hyperammonemia and predisposing susceptibility to the metabolic insult. McKinney et $\mathrm{al}^{22}$ believed that acute HE occurs along a spectrum, with multifocal diffuse cortical findings being at the severe end.

\section{Brain Edema in Chronic HE}

Structural MR Imaging. In chronic HE (type C), low-grade brain edema has been reported in many human and animal studies by using other advanced MR images, ${ }^{2-7}$ but no mild abnormalities can be detected in conventional T1- and T2-weighted images, except basal ganglia hyperintensity, ${ }^{1}$ which is attributed to hypermagnesemia (Fig $2 A,-B$ ). Some investigators also reported high signal intensity along the hemispheric white matter in or around the corticospinal tract on fast FLAIR T2-weighted images (Fig $2 C$ ), strikingly similar to signal intensity abnormalities noted in cases of amyotrophic lateral sclerosis, a neurodegenerative disease that affects motor neurons. The progressive normalization of the high signal intensity abnormalities on the FLAIR sequence in patients with cirrhosis can be found after successful liver transplantation or effective treatments, which is different from cerebral infarct. ${ }^{1}$ Observation of white matter high intensity in FLAIR images alone without confirmation with additional imaging sequences cannot be used to interpret these changes in patients with HE.

MTI. MTI is a useful technique to detect low-grade brain edema in HE. ${ }^{1,4,23}$ It is mainly based on the interaction (cross-relaxation) between protons in a relatively free environment (bulk water) and those in which motion is restricted (immobile water). MTI can allow the measurement of MTRs, which can reflect brain parenchymal changes that may not be visible by using standard MR imaging techniques. ${ }^{6}$ All studies by using MTI in HE found reduced MTR values in all the examined regions of the brain in HE 


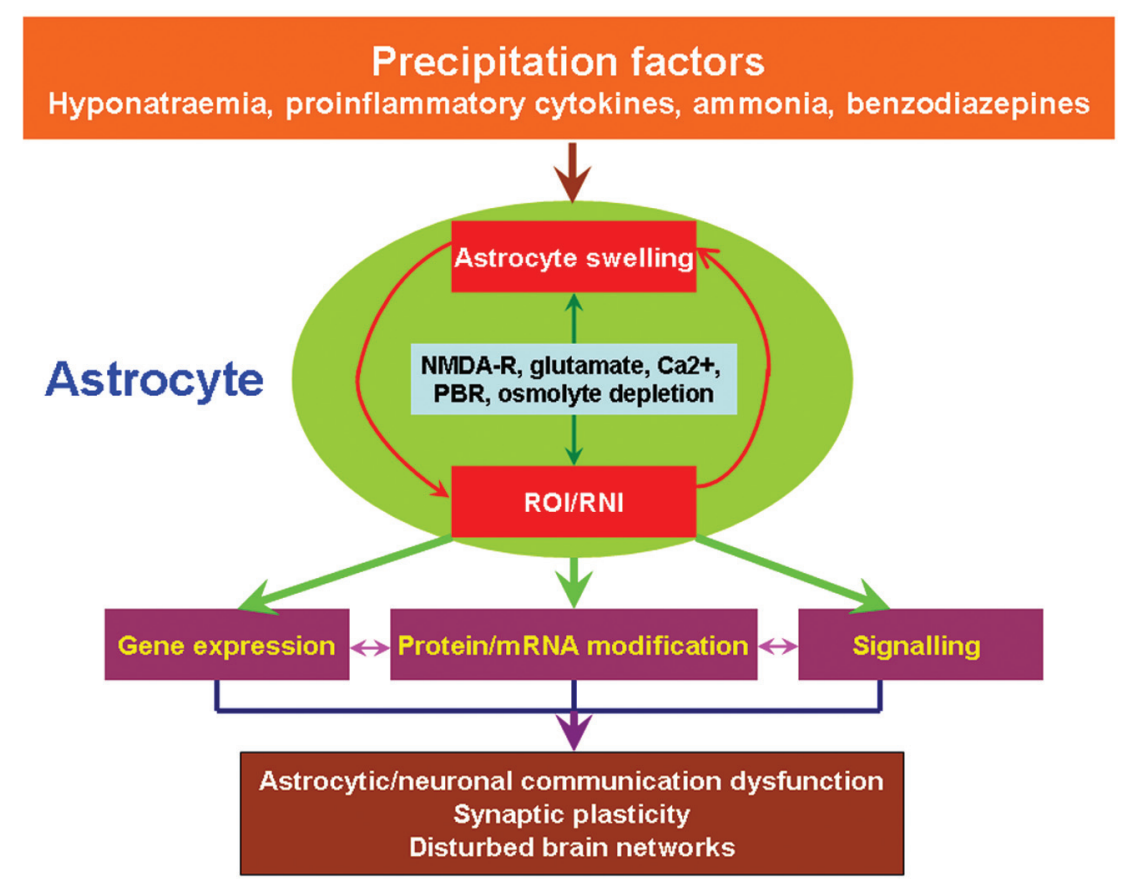

FIG 1. Low-grade brain edema in HE. Ammonia induces astrocyte swelling, which is, in part, counteracted by osmolyte depletion but can be aggravated by a set of precipitating factors. Astrocyte swelling involves activation of the $N$-methyl-D-aspartate receptor and the generation of reactive nitrogen oxide species, which again cause astrocyte swelling. The autoamplificatory loop produces signals that change the astrocyte function at multiple levels, including covalent modifications of proteins and ribonucleic acid, resulting in impaired glioneuronal communication, synaptic plasticity, and brain networks, which finally account for the symptoms of HE. Adapted with permission from Haussinger et al, ${ }^{18}$ with partial revision, and from BMJ Publishing Group Ltd. PBR indicates peripheral benzodiazepine receptor; RNI, reactive nitrogen intermediates; $\mathrm{ROI}$, reactive oxygen intermediates.
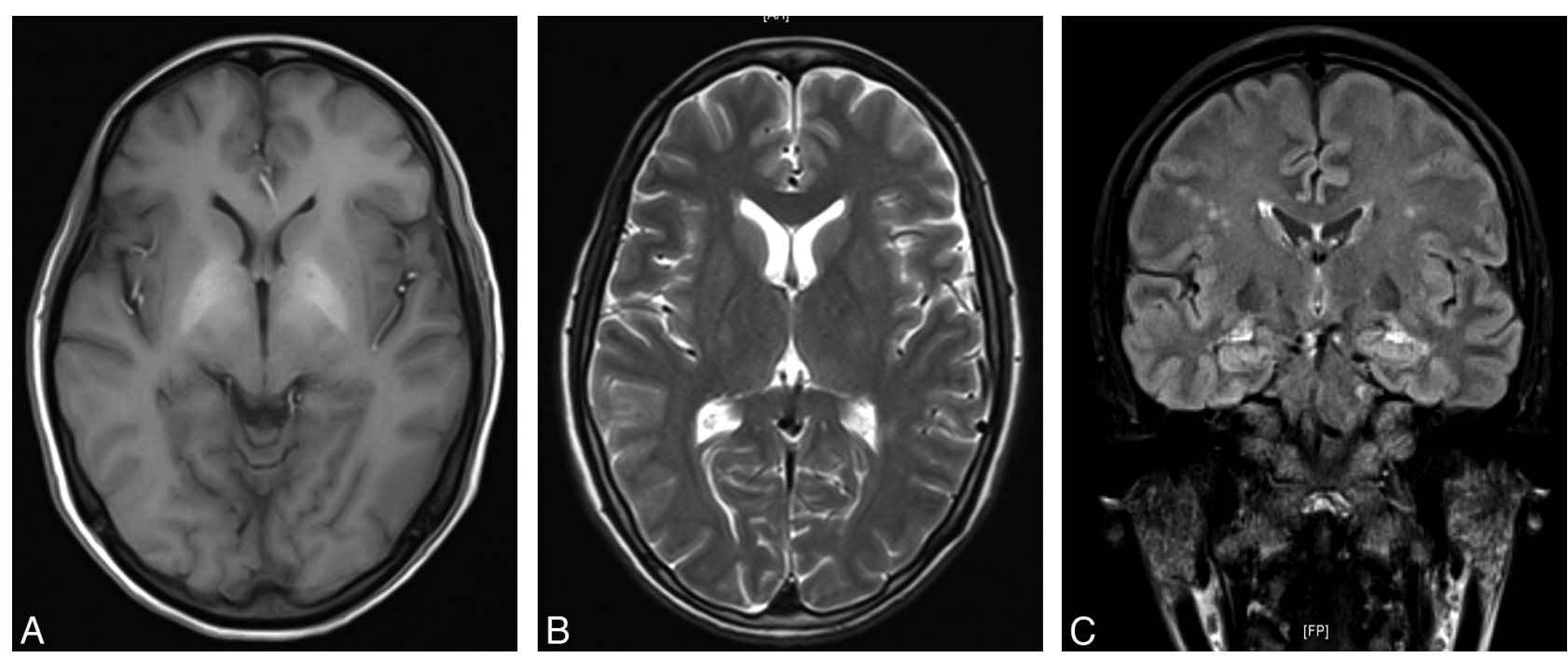

FIG 2. Structural MR imaging findings in patients with cirrhosis. A, Axial T7-weighted image at the level of the basal ganglia shows symmetric high intensity in the bilateral basal ganglia. B, Corresponding axial T2-weighted image does not show abnormal findings. C, Coronal T2 FLAIR image shows multiple high signal intensities in or around the corticospinal tract.

compared with the controls (Fig 3). Lower MTR may result from pathologies that alter the structural integrity and the relative macromolecular-water composition of brain parenchyma, such as in multiple sclerosis plaques or in end-stage cirrhosis. Compared with other diseases, such as experimental autoimmune encephalomyelitis, toxic demyelination, progressive multifocal leukoencephalopathy, human immunodeficiency virus encephalitis, and multiple sclerosis, the MTR decrease in chronic HE is mild (approximately $10 \%$ ) and is not accompanied by significant abnor- malities on conventional T1- and T2-weighted images. ${ }^{23}$ This MTR decrease almost returns to normal values after liver transplantation. ${ }^{1}$ Thus, the phenomenon observed on MTI may support the hypothesis that mild brain edema is present in patients with cirrhosis. $^{24}$

DWI. DWI is a method for quantifying the movement of water molecules. Water molecules follow the principles of Brownian motion. In a container of water, molecules undergo free thermally 

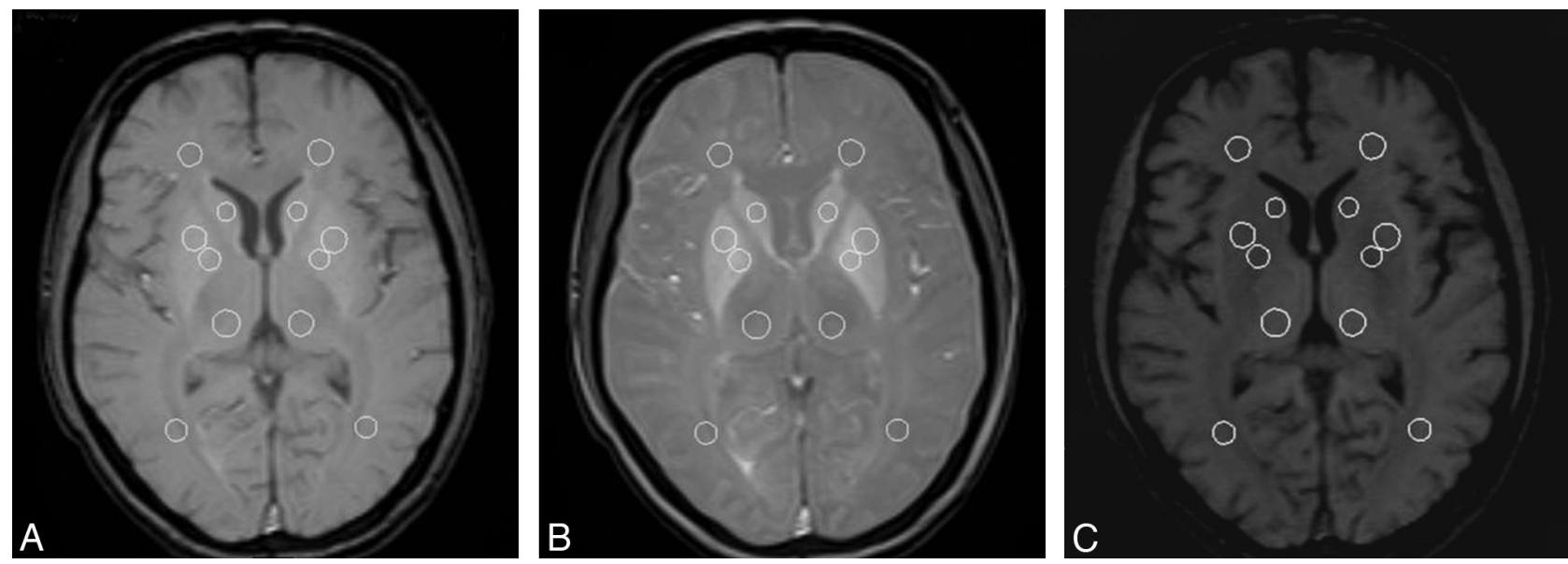

FIG 3. MTI in a 37-year-old male patient with cirrhosis. $A$, MR image without the magnetic transfer saturation pulse. $B$, MR image with the magnetic transfer saturation pulse. $C$, MR image resulting from $B$ minus $A$, in which changes of signal intensity reflect the signal intensity suppressed by magnetic transfer saturation pulse, correlating with macromolecule protein content in tissue. Some regions of interest for quantification of MTR are seen in these images.
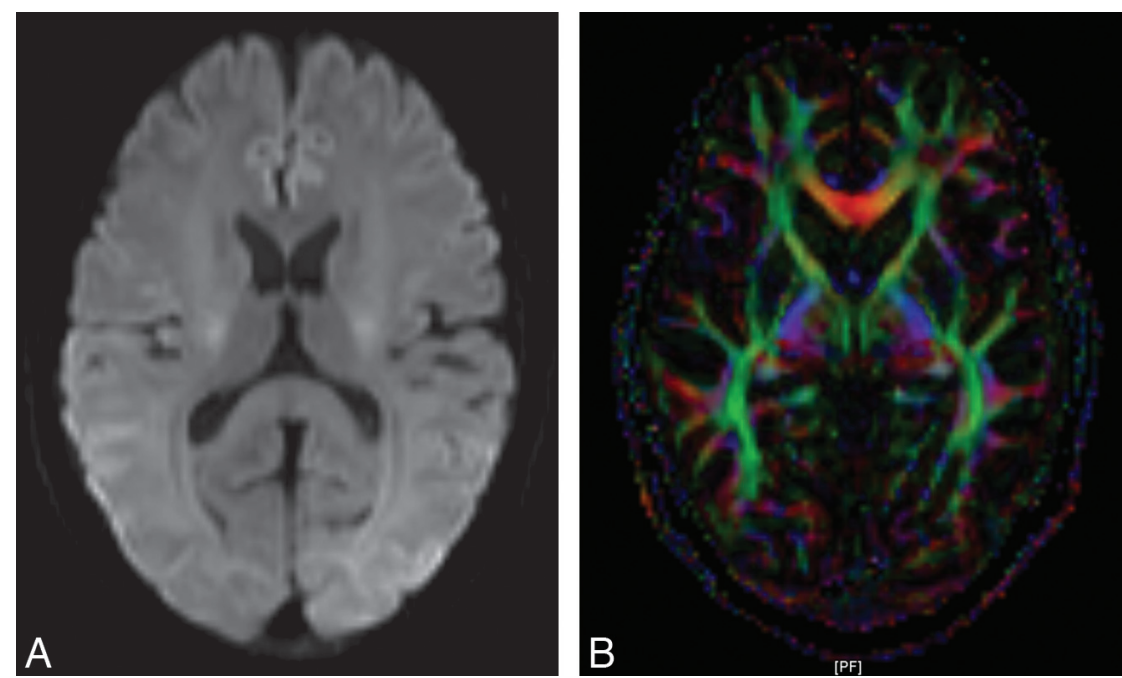

FIG 4. DTI in 1 patient with cirrhosis, acquired with $3 T$ MR imaging. DWI $(A)$ and FA image $(B)$, in which green represents fiber tracts along the anteroposterior; blue, caudocranial; and red, transverse direction.

agitated diffusion, which is also called isotropic (meaning equal in all directions). However, limited (anisotropic) diffusion is observed in the brain because the movement of water molecules is restricted by their interactions with cell membranes and macromolecules. DWI derives its image contrast on the basis of differences in the mobility of protons (primarily associated with water) between tissues. Using DWI, one can calculate a diffusivity value to quantitatively assess the strength of water molecule movement in tissue.

DTI. DTI can be used to obtain anisotropy information of water molecule diffusion in tissues by applying specific diffusion-weighted field gradients in at least 6 directions. The most commonly used indices for the measurement of anisotropic diffusions by DTI include the relative anisotropy, FA, and the volume ratio indices. These indices provide quantitative measurements of the changes of white matter integrity in different brain regions that are affected by diseases. Using DTI techniques together with advanced fiber tracking algorithms, which are mathematic approaches to derive white matter tractography from DTI data, one can construct 3D trajectories of neural tracts noninvasively, allowing the modeling of white matter neural connectivity.

Recently, DWI and DTI have been used to detect brain abnormalities in patients with $\mathrm{HE}$ (Fig 4). ${ }^{20,25,26}$ In this setting, $\mathrm{ADC} / \mathrm{mean}$ diffusivity was a reliable tool for quantification of low-grade HE and could predict the development of overt HE. ${ }^{26}$ The mean diffusivity values were increased in patients with cirrhosis with MHE compared with patients with no HE or controls. Patients with MHE could be separated from patients with no HE with a sensitivity of $70 \%-90 \%$ and a specificity of $85 \%-90 \%$ by using DWI. In addition, diffusivity values of white matter, such as the frontal and parietal white matter, were found to be predictive of further bouts of overt HE. ${ }^{25}$

DWI and DTI have the potential to define the nature of lowgrade brain edema in patients with cirrhosis. However, recent studies on low-grade brain edema with DWI/DTI techniques have 

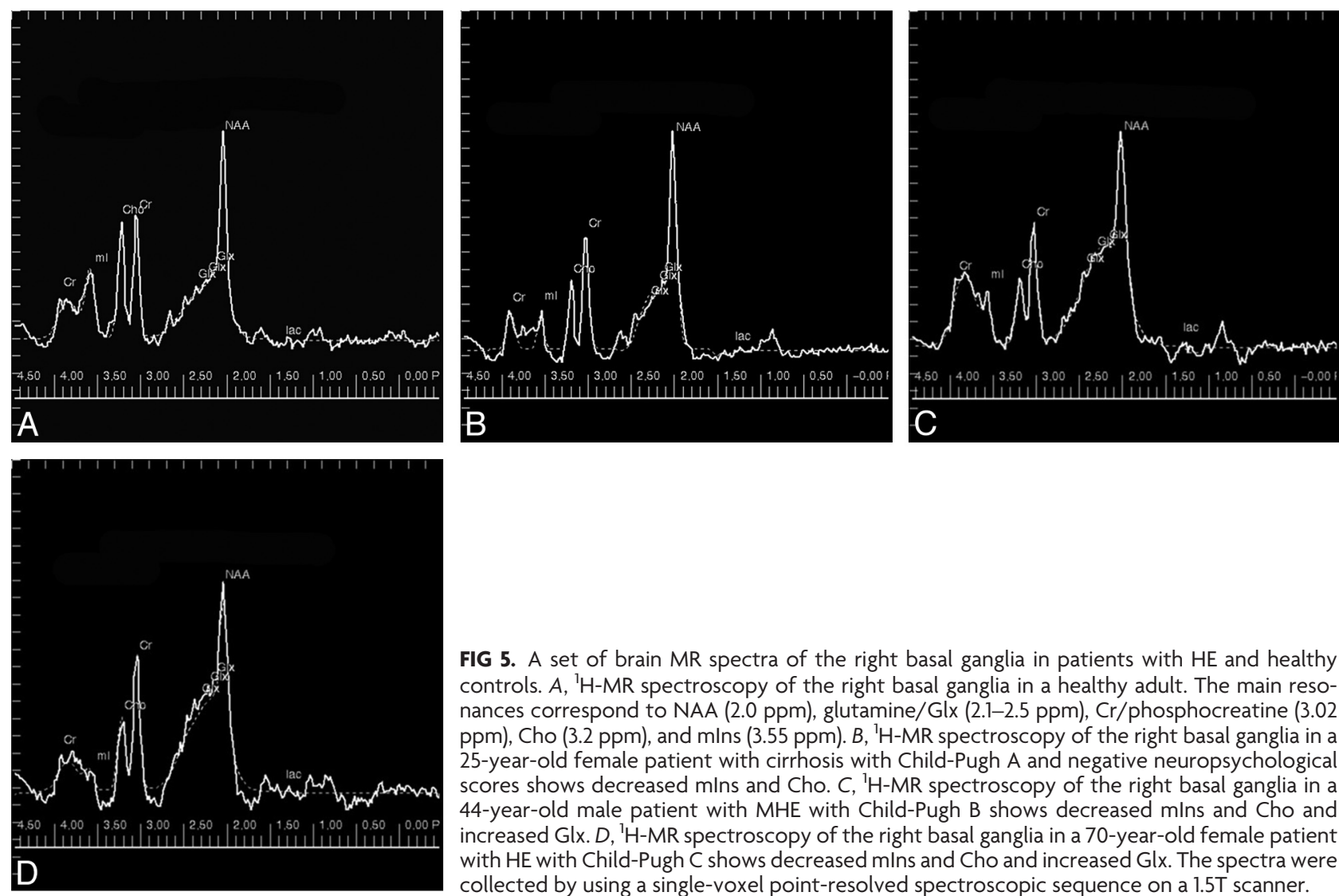

FIG 5. A set of brain MR spectra of the right basal ganglia in patients with $\mathrm{HE}$ and healthy controls. A, 'H-MR spectroscopy of the right basal ganglia in a healthy adult. The main resonances correspond to NAA (2.0 ppm), glutamine/Glx (2.1-2.5 ppm), Cr/phosphocreatine (3.02 $\mathrm{ppm})$, Cho (3.2 ppm), and mlns (3.55 ppm). $B,{ }^{1} \mathrm{H}-\mathrm{MR}$ spectroscopy of the right basal ganglia in a 25-year-old female patient with cirrhosis with Child-Pugh $A$ and negative neuropsychological scores shows decreased mlns and Cho. ${ }^{1},{ }^{1} \mathrm{H}-\mathrm{MR}$ spectroscopy of the right basal ganglia in a 44-year-old male patient with MHE with Child-Pugh B shows decreased mlns and Cho and increased Glx. D, ${ }^{1} \mathrm{H}$-MR spectroscopy of the right basal ganglia in a 70-year-old female patient with HE with Child-Pugh C shows decreased $\mathrm{mlns}$ and $\mathrm{Cho}$ and increased Glx. The spectra were collected by using a single-voxel point-resolved spectroscopic sequence on a 1.5T scanner.

not reached consistent results to support which DWI/DTI index has a predominant role in the pathophysiology of HE, partly because of different types of HE models, subjects, and techniques used in different studies. ${ }^{7,27-30}$ Some studies on the use of mean diffusivity measurements in DTI within normal-appearing white matter of patients with cirrhosis have shown a significant increase in brain water diffusivity, which was more pronounced with the increasing grade of HE. ${ }^{23,27}$ These diffusivity values correlated with neuropsychological impairment and serum venous ammonia. These findings probably reflect an accumulation of water in the extracellular compartment and, therefore, do not support the hypothesis of astrocytic swelling as the cause of diffuse brain edema in chronic liver failure. ${ }^{1}$ In the study of Kale et al, ${ }^{27}$ significantly increased mean diffusivity was found with insignificant changes in FA in various regions of brain in patients with MHE or type C HE compared with controls, indicating an increase in interstitial water in the brain parenchyma without any microstructural changes. The study of Nath et $\mathrm{al}^{28}$ found that the presence of significantly decreased FA and linear anisotropy and increased spheric isotropy along with no significant change in mean diffusivity and planar anisotropy suggests the presence of both intracellular and extracellular components of cerebral edema in patients with acute-on-chronic liver failure. Decreased mean diffusivity values in patients with fulminant hepatic failure suggest that predominant cytotoxic edema may be present. ${ }^{29}$

The study of Chavarria et $\mathrm{al}^{7}$ in a rat model of acute liver failure supported the cytotoxic origin of brain edema, suggesting that the metabolism of ammonia in astrocytes induces an increase of glutamine and lactate, which appears to mediate cellular swelling. Rai et $\mathrm{al}^{30}$ reported that decreased mean diffusivity and increased spheric isotropy in patients with acute hepatic failure associated with decreased FA represent cytotoxic and interstitial components of brain edema, respectively. Thus, it appears that 2 types of brain edema may simultaneously exist in liver failure, predominantly intracellular in acute forms, interstitial in chronic forms, and both in acute-on-chronic liver failure.

MR Spectroscopy. MR spectroscopy is a noninvasive analytic method often paired with MR imaging in neuroradiology practices. It is capable of identifying and measuring the individual chemicals in the brain regions localized and sampled from MR images, offering the potential to interrogate the molecular process within tissues and fluids in vivo and in vitro. Two nuclei $\left(\mathrm{ie},{ }^{1} \mathrm{H}\right.$ and ${ }^{36} \mathrm{P}$ ) can be used for clinical MR spectroscopy studies. ${ }^{5,6}$ ${ }^{1} \mathrm{H}$-MR spectroscopy is widely used in clinical practice to provide information on brain metabolites such as Cho, Cr, NAA, glutamine, and Glx, as well as osmolytes, such as mIns and taurine.

${ }^{1} \mathrm{H}$-MR spectroscopy is widely used in HE studies and has consensus findings on the intracellular metabolite changes in HE, while ${ }^{31} \mathrm{P}$ MR spectroscopy studies are relatively rare and no consensus results are available. ${ }^{5,6}$ Typical ${ }^{1} \mathrm{H}$-MR spectroscopy findings of $\mathrm{HE}$ are lower $\mathrm{Cho} / \mathrm{Cr}$ and $\mathrm{mIns} / \mathrm{Cr}$ and higher $\mathrm{Glx} / \mathrm{Cr}$, 5,6,31 which are seen in all examined brain regions of patients compared with controls (Fig 5). Hyperammonia can account for the MR spectroscopy findings in patients with cirrhosis. One recent study $^{32}$ of induced hyperammonemia in patients with cirrhosis showed that ammonia could directly drive changes in brain water distribution as a mechanism for cerebral edema development. In 
the theory of hyperammonemia accounting for the pathogenetic mechanism of HE, the increase in brain glutamine (increased Glx/Cr on ${ }^{1} \mathrm{H}$-MR spectroscopy) due to hyperammonemia during HE increases intracellular osmolality. To maintain osmotic equilibrium, the astrocytes lose osmolytes such as mIns and Cho (decreased mIns/Cr and Cho/Cr on ${ }^{1} \mathrm{H}$-MR spectroscopy) and a large amount of water enters the astrocyte and results in cellular swelling and impairment of cellular metabolism, further influencing neuronal and astrocyte function and interaction between them. ${ }^{1,5,6}$

Patients without cirrhosis may tolerate such precipitating factors without developing HE symptoms because their osmolyte systems for counteraction of cell swelling are not exhausted. However, organic osmolytes are largely depleted in patients with cirrhosis to compensate for glial glutamine accumulation, and there may be little room for action of these volume-regulatory mechanisms against further challenges of cell volume. This labile situation may explain not only the rapid kinetics of HE episodes but also the occasional appearance of clinically overt cerebral edema in end-stage cirrhosis. ${ }^{18}$ With ${ }^{1} \mathrm{H}-\mathrm{MR}$ spectroscopy, no significant changes of NAA were detected in patients with cirrhosis compared with healthy subjects, which indicates the integrity of neurons. ${ }^{31}$

However, this finding appears to be contradictory to the evidence of brain atrophy from voxel-based morphometry. ${ }^{33}$ It is possible that mild-to-moderate loss of myelin and axons is not always found in chronic HE. ${ }^{2}$ Of these ${ }^{1} \mathrm{H}-\mathrm{MR}$ spectroscopy parameters, mIns seems to be a more sensitive biomarker than Cho to detect HE. ${ }^{18,31,34}$ mIns levels were related to the Child-Pugh score and severity of HE. ${ }^{31,34}$ However, cutoff values have not been established, and the diagnostic accuracy of ${ }^{1} \mathrm{H}$-MR spectroscopy remains uncertain. ${ }^{1} \mathrm{H}$-MR spectroscopy is unable to differentiate some metabolites, such as Glx and taurine. 2D ${ }^{1} \mathrm{H}-\mathrm{MR}$ spectroscopy has enhanced spectral resolution to show more metabolites, which can be helpful in understanding the pathogenesis of HE. Wide availability of 3T MR imaging scanners can improve the ability of ${ }^{1} \mathrm{H}$-MR spectroscopy to differentiate Glx.

${ }^{1} \mathrm{H}-\mathrm{MR}$ spectroscopy findings also supported the role of multiple precipitating factors, such as hyperammonia and proinflammatory cytokines in the pathogenesis of HE. Gupta et $\mathrm{al}^{10}$ found that proinflammatory cytokines showed positive correlation with spheric anisotropy and negative correlation with mean diffusivity in various brain regions, including the spectroscopy voxel in patients with acute liver failure. These correlations suggest that both DTI-derived metrics and ${ }^{1} \mathrm{H}-\mathrm{MR}$ spectroscopy measure the synergistic effect of hyperammonemia and proinflammatory cytokines and may be used as noninvasive tools for understanding the pathogenesis of brain edema in acute liver failure.

${ }^{1} \mathrm{H}$-MR spectroscopy is also a useful tool to monitor the metabolic changes after treatment or interventions for HE. ${ }^{1} \mathrm{H}-\mathrm{MR}$ spectroscopy abnormalities are reversible after successful liver transplantation and effective treatment, ${ }^{35}$ indicating the nature of reversible brain edema in patients with cirrhosis. ${ }^{1,20,21}$ The evolution of the metabolic changes differs for each metabolite after liver transplantation. The Cho peak normalizes earlier than the other peaks, and the glutamine/Glx peak reaches the normal range at 1-2 months. The peak mIns abnormalities may take 3-7 months to reach normal values. This reversibility of ${ }^{1} \mathrm{H}-\mathrm{MR}$ spectroscopy abnormalities precedes the disappearance of pallidal hyperintensity after liver transplantation and correlates with improvements in the neurologic manifestations of $\mathrm{HE}^{32,35}$ However, ${ }^{1} \mathrm{H}-\mathrm{MR}$ spectroscopy abnormalities can also be detected in patients with abnormal liver function after liver transplantation.

BOLD fMRI. Although no clinically overt brain edema and intracranial pressure can be detected with conventional T1- and T2weighted imaging, small increases in astrocyte water content, present in cirrhosis with MHE or overt HE, may have important functional consequences through triggering of a set of molecular events. ${ }^{18}$ Disturbance of oscillatory networks detected in patients with low-grade $\mathrm{HE}$ at magnetoencephalography supports the speculation that glioneuronal communication resulting from the low-grade brain edema is impaired in these patients. ${ }^{36}$ Many behavioral studies have claimed the existence of neuropsychological abnormalities, such as attention and fine-motor alterations, in patients with cirrhosis without overt HE. ${ }^{13,14}$ Thus, it is rational and feasible to investigate brain function changes in patients with cirrhosis.

Recently, some investigators demonstrated an abnormal brain network in patients with cirrhosis by using BOLD fMRI, even to predict the development of overt HE. fMRI detects BOLD changes in MR imaging signal intensity that arise when changes in neuronal activity occur following a change in brain state-for example, responding to a stimulus or performing a specific task. The BOLD MR imaging signal-intensity change is the result of combined changes of hemoglobin oxygenation and cerebral blood flow during brain activation. With 3D imaging and high spatial resolution capabilities, fMRI has been developed as a primary functional brain-mapping tool, offering a wide range of applications in both clinical (such as neurosurgery preoperative planning) and basic neurosciences. ${ }^{37}$

fMRI can be classified as either task-related or resting-state fMRI, according to experimental paradigms. In a task-related fMRI design, a subject is placed in the magnet and scanned while various different kinds of stimulus are administered in a controlled fashion. Two main experimental paradigms are in common use in task-related fMRI studies: block design and eventrelated paradigms. ${ }^{37}$ In resting-state fMRI, the participants are required to rest with their eyes closed and keep their heads still and not to think of anything in particular during MR imaging scans lasting typically a few minutes.

Despite increasing use of fMRI in the past decade, fMRI studies in patients with cirrhosis are few, even though it has become increasingly attractive in recent years. A few task-related fMRI studies have focused on the attention-function impairment in patients with cirrhosis with different task stimuli. For example, Zafiris et $\mathrm{al}^{38}$ first analyzed the pathologically impaired neural mechanisms of patients with cirrhosis by using fMRI. They found decreased judgment-related BOLD activation in the right IPL, impaired neural interaction between the IPL and the parieto-occipital cortex, the intraparietal sulcus, the anterior cingulate cortex, the right prefrontal cortex, the medial temporal lobe, and the extrastriate cortex V5 and an enhanced coupling between the IPL 

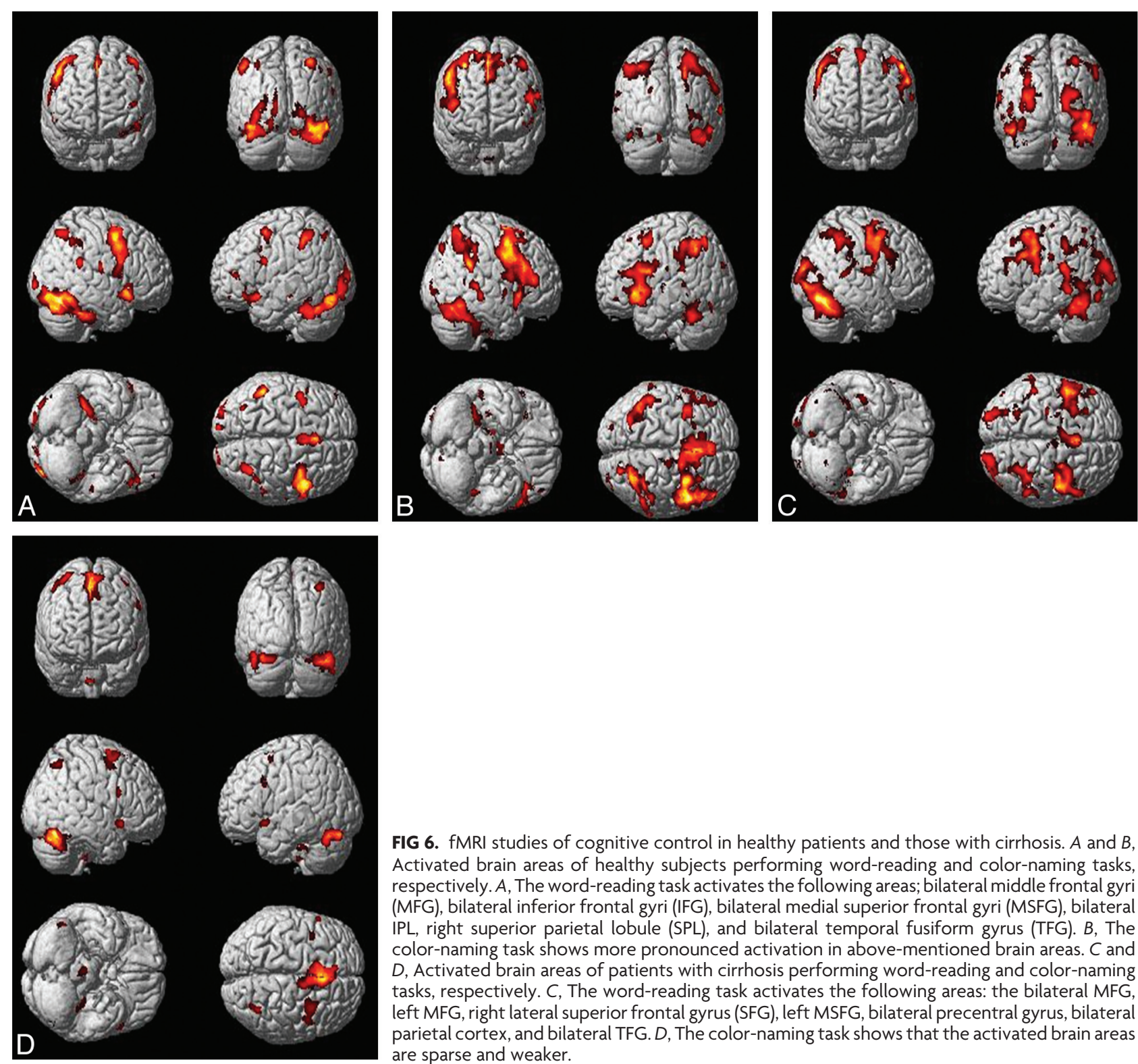

FIG 6. fMRI studies of cognitive control in healthy patients and those with cirrhosis. $A$ and $B$, Activated brain areas of healthy subjects performing word-reading and color-naming tasks, respectively. $A$, The word-reading task activates the following areas; bilateral middle frontal gyri (MFG), bilateral inferior frontal gyri (IFG), bilateral medial superior frontal gyri (MSFG), bilateral IPL, right superior parietal lobule (SPL), and bilateral temporal fusiform gyrus (TFG). B, The color-naming task shows more pronounced activation in above-mentioned brain areas. $C$ and $D$, Activated brain areas of patients with cirrhosis performing word-reading and color-naming tasks, respectively. C, The word-reading task activates the following areas: the bilateral MFG, left MFG, right lateral superior frontal gyrus (SFG), left MSFG, bilateral precentral gyrus, bilateral parietal cortex, and bilateral TFG. $D$, The color-naming task shows that the activated brain areas are sparse and weaker.

and the postcentral cortex in patients with MHE compared with controls. This study demonstrated an early impaired and compensatory neural mechanism during visual judgment already in the earliest stages of HE. Zhang et $\mathrm{al}^{14}$ used a Chinese character Stroop task as the target stimulus to investigate the neural mechanism of cognitive control impairment in patients with cirrhosis by using the fMRI method, providing further evidence in support of the fact that patients with cirrhosis have impaired cognitive control function and that the abnormal anterior cingulate cortexprefrontal cortex-parietal "fusiform" cortex circuit could be the neural mechanism responsible for this impaired cognitive control (Fig 6). However, paradigms should be kept simple to isolate specific brain areas in the task-induced fMRI. Thus, the technique is only suitable for patients who can perform tasks and therefore is better suited to low-grade or minimal HE. ${ }^{7}$ To the best of our knowledge, no more task-related fMRI studies on HE have been published to date.

Recently, increasing attention has been focused on detecting brain activities in the resting state. Currently, the spatial topogra- phies of brain functional connectivity at the resting state are widely known as resting-state networks. Resting-state networks normally represent a remarkably robust phenomenon, which is present in rudimentary form early in human life and later reorganized as brain development proceeds through childhood. These networks persist during task performance and sleep and under sedation. ${ }^{39}$ Most reported resting-state networks include the DMN, the sensorimotor component, the executive control component, up to 3 visual components, 2 lateralized frontoparietal components, the auditory component, and the temporoparietal component. ${ }^{40}$ As already reported, these resting-state networks consist of anatomically separated but functionally connected regions displaying a high level of correlated BOLD signal-intensity activity. Currently, the most studied network is the DMN, including the medial prefrontal cortex, rostral anterior cingulate, PCC, and precuneus. What the DMN represents is unclear, but it is known to have high metabolic activity during rest and is suppressed during cognitively demanding tasks, such as visual and auditory attention, language processing, memory, and motor ac- 

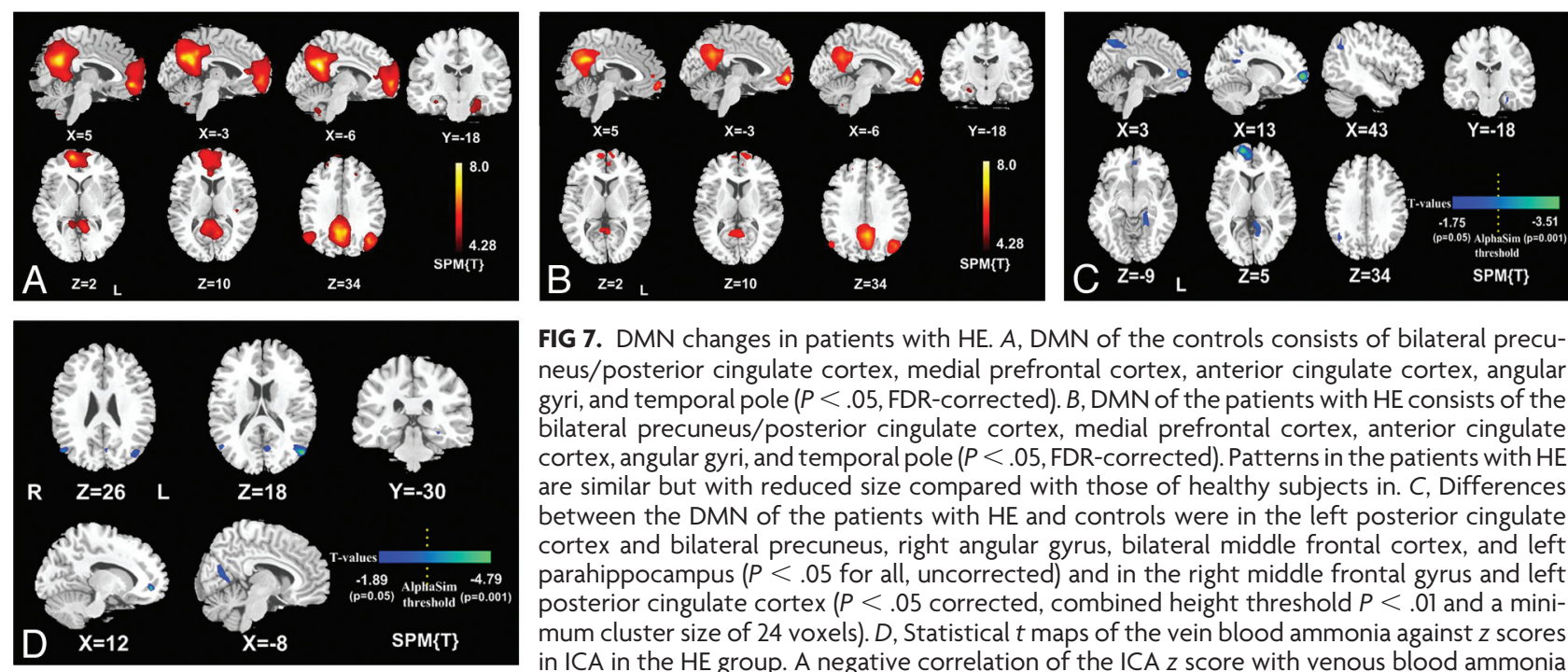

FIG 7. DMN changes in patients with HE. A, DMN of the controls consists of bilateral precuneus/posterior cingulate cortex, medial prefrontal cortex, anterior cingulate cortex, angular gyri, and temporal pole ( $P<.05$, FDR-corrected). $B, \mathrm{DMN}$ of the patients with $\mathrm{HE}$ consists of the bilateral precuneus/posterior cingulate cortex, medial prefrontal cortex, anterior cingulate cortex, angular gyri, and temporal pole $(P<.05$, FDR-corrected). Patterns in the patients with HE are similar but with reduced size compared with those of healthy subjects in. $C$, Differences between the DMN of the patients with $\mathrm{HE}$ and controls were in the left posterior cingulate cortex and bilateral precuneus, right angular gyrus, bilateral middle frontal cortex, and left parahippocampus $(P<.05$ for all, uncorrected) and in the right middle frontal gyrus and left posterior cingulate cortex $(P<.05$ corrected, combined height threshold $P<.01$ and a minimum cluster size of 24 voxels). $D$, Statistical $t$ maps of the vein blood ammonia against $z$ scores in ICA in the HE group. A negative correlation of the ICA $z$ score with venous blood ammonia was found in the right middle frontal gyrus, left posterior cingulate cortex, left parahippocampus, bilateral angular gyri $(P<.05$ for all, uncorrected), and the left angular gyrus color-coded bright blue $(P<.05$ corrected, combined height threshold $P<.01$ and a minimum cluster size of 24 voxels).

tivity. An abnormal default function network has been known to relate to Alzheimer disease, autism, attention deficit/hyperactivity disorder, schizophrenia, epilepsy, and other diseases. In a study by Zhang et al, ${ }^{41}$ the DMN of patients with cirrhosis was investigated with the subtraction method by using a blocked-design fMRI in which a block design was used with a modified Chinese Stroop task as the target stimulus. In that study, the subtraction method was used to study the resting-state network in patients with hepatic cirrhosis; the functional and behavioral data suggest that patients with cirrhosis may have an abnormal deactivation mode. The absence of deactivation in the PCC and precuneus may be a sensitive rather than specific marker in patients with hepatic cirrhosis.

Recently, in a standard resting-state fMRI study including 10 patients with $\mathrm{HE}$ and 14 controls, Zhang et $\mathrm{al}^{42}$ found significantly reduced functional connectivity in the right middle frontal gyrus, left precuneus, and left PCC in the patients with HE (Fig $7 A-C) . Z$ scores of the left angular gyrus and left PCC were found to have negative correlation with venous blood ammonia levels, an important biomarker for HE, in the HE group (Fig 7D). Our recent study further demonstrated that patients with HE had diffuse abnormalities in intrinsic brain activity with ALFF, based on whole-brain functional analysis algorithms. The level of decreased ALFF in the DMN and increased ALFF in the posterior insular cortex depends on HE severity, suggesting continuous impairment of the DMN and a compensatory role of the insula during the progression of HE (Qi RF, November 12, 2011, unpublished data). Thus, it appears that resting-state fMRI with ALFF analysis may be an effective and noninvasive technique to predict the development of HE. To the best of our knowledge, no fMRI studies on other resting-state networks in patients with HE have been published at the time of this writing. However, it is possible that the diffusely abnormal resting-state brain networks are present in patients with MHE and chronic HE on the basis of the above-mentioned fMRI studies. ${ }^{41,42}$

\section{PERSPECTIVE}

In recent years, the understanding of the pathophysiology of $\mathrm{HE}$ has been improved with advanced MR imaging techniques, especially of the role of low-grade brain edema in the pathophysiology of HE; however, many issues remain unresolved. We need to define the nature of low-grade brain edema in different types of HE, such as chronic, acute, and acute-on-chronic. Perfusion-weighted MR imaging, such as arterial spin-labeling, a noninvasive MR imaging technique in which the blood is used as an endogenous contrast agent, can be used to investigate the hemodynamic changes of $\mathrm{HE}$ and to differentiate patients with cirrhosis from patients with MHE. BOLD-based AMRI should also be further investigated; the role of BOLD fMRI in the neural basis of cognitive and functional impairment of patients with $\mathrm{HE}$ and of the progression of HE from cirrhosis to minimal HE to overt HE should be clarified. The molecular imaging technique, combined MR imaging/PET, may have a promising role in uncovering the pathogenesis of $\mathrm{HE}$, especially the role of molecular markers (such as peripheral-type benzodiazepine receptors) in HE. A large-cohort well-designed clinical trial should be performed in the future to demonstrate the role of multimodality MR imaging techniques in the early detection and follow-up of HE.

\section{CONCLUSIONS}

HE appears as diffuse mild brain edema and is associated with functional changes, such as those in attention and default mode brain areas, which have been demonstrated by a variety of MR imaging techniques. MR imaging offers a range of capabilities for investigating $\mathrm{HE}$ and is helpful in clarifying the pathophysiologic mechanism of HE. It will play an increasingly important role in early diagnosis of HE with more MR imaging studies performed in this field. The recent development of fMRI and DTI provides and expands the applications of the MR imaging study of HE with the capability of assessing functional changes in brain affected by $\mathrm{HE}$, therefore, providing the opportunity to uncover the pathophysiologic mechanisms of HE. 


\section{ACKNOWLEDGMENTS}

We thank Dr. Philip K. Liu at Athinoula A. Martinos Center for Biomedical Imaging, Department of Radiology, Massachusetts General Hospital, and Harvard Medical School, for reviewing the manuscript, and Dr. Shengyong Wu for providing critical comments.

\section{REFERENCES}

1. Rovira A, Alonso J, Cordoba J. MR imaging findings in hepatic encephalopathy. AJNR Am J Neroradiol 2008;29:1612-21

2. Matsusue E, Kinoshita T, Ohama E, et al. Cerebral cortical and white matter lesions in chronic hepatic encephalopathy: MR-pathologic correlations. AJNR Am J Neuroradiol 2005;26:347-51

3. Córdoba J. New assessment of hepatic encephalopathy. J Hepatol 2011;54:1030-40

4. Poveda MJ, Bernabeu A, Concepción L, et al. Brain edema dynamics in patients with overt hepatic encephalopathy: a magnetic resonance imaging study. Neuroimage 2010;52:481-87

5. McPhail MJ, Taylor-Robinson SD. The role of magnetic resonance imaging and spectroscopy in hepatic encephalopathy. Metab Brain Dis 2010;25:65-72

6. Grover VP, Dresner MA, Forton DM, et al. Current and future applications of magnetic resonance imaging and spectroscopy of the brain in hepatic encephalopathy. World J Gastroenterol 2006; 12:2969-78

7. Chavarria L, Oria M, Romero-Gimenez J, et al. Diffusion tensor imaging supports the cytotoxic origin of brain edema in a rat model of acute liver failure. Gastroenterology 2010;138:1566-73

8. Buterworth RF. Hepatic encephalopathy: a central neuroinflammatory disorder? Hepatology 2011;53:1372-76

9. Rodrigo R, Cauli O, Gomez-Pinedo U, et al. Hyperammonemia induces neuroinflammation that contributes to cognitive impairment in rats with hepatic encephalopathy. Gastroenterology 2010;139:675-84

10. Gupta RK, Yadav SK, Rangan M, et al. Serum proinflammatory cytokines correlate with diffusion tensor imaging derived metrics and (1)H-MR spectroscopy in patients with acute liver failure. Metab Brain Dis 2010;25:355-61

11. Ferenci P, Lockwood A, Mullen K, et al. Hepatic encephalopathy: definition, nomenclature, diagnosis, and quantification-final report of the working party at the 11th World Congresses of Gastroenterology, Vienna, 1998. Hepatology 2002;35:716-21

12. Yadav SK, Saksena S, Srivastava A, et al. Brain MR imaging and $1 \mathrm{H}-\mathrm{MR}$ spectroscopy changes in patients with extrahepatic portal vein obstruction from early childhood to adulthood. AJNR Am J Neuroradiol 2010;31:1337-42

13. Randolph C, Hilsabeck R, Kato A, et al. Neuropsychological assessment of hepatic encephalopathy: ISHEN practice guidelines. Liver Int 2009;29:629-35. Epub 2009 Mar 19

14. Zhang LJ, Yang G, Yin J, et al. Neural mechanism of cognitive control impairment in patients with hepatic cirrhosis: a functional magnetic resonance imaging study. Acta Radiol 2007;48:577-87

15. Romero-Gómez M, Córdoba J, Jover R, et al. Value of the critical flicker frequency in patients with minimal hepatic encephalopathy. Hepatology 2007;45:879-85

16. Kreis R, Ross BD, Farrow NA, et al. Metabolic disorders of the brain in chronic hepatic encephalopathy detected with H-1 MR spectroscopy. Radiology 1992;182:19-27

17. Zemtsova I, Görg B, Keitel V, et al. Microglia activation in hepatic encephalopathy in rats and humans. Hepatology 2011;54:204-15

18. Häussinger D, Schliess F. Pathogenetic mechanisms of hepatic encephalopathy. Gut 2008;57:1156-65

19. Arnold SM, Els T, Spreer J, et al. Acute hepatic encephalopathy with diffuse cortical lesions. Neuroradiology 2001;43:551-54

20. McKinney AM, Lohman BD, Sarikaya B, et al. Acute hepatic encephalopathy: diffusion-weighted and fluid-attenuated inversion recovery findings, and correlation with plasma ammonia level and clinical outcome. AJNR Am J Neuroradiol 2010;31:1471-79
21. U-King-Im JM, Yu E, Bartlett E, et al. Acute hyperammonemic encephalopathy in adults: imaging findings. AJNR Am J Neuroradiol 2011;32:413-18

22. McKinney AM, Sarikaya B, Spanbauer J, et al. Acute hepatic (or hyperammonemic) encephalopathy: diffuse cortical injury and the significance of ammonia. AJNR Am J Neuroradiol 2011;32:E142

23. Miese F, Kircheis G, Wittsack HJ, et al. 1H-MR spectroscopy, magnetization transfer, and diffusion-weighted imaging in alcoholic and nonalcoholic patients with cirrhosis with hepatic encephalopathy. AJNR Am J Neuroradiol 2006;27:1019-26

24. Rovira A, Córdoba J, Raguer N, et al. Magnetic resonance imaging measurement of brain edema in patients with liver disease: resolution after transplantation. Curr Opin Neurol 2002;15:731-37

25. Barbiroli B. Diffusion MRI shows increased water apparent diffusion coefficient in the brains of cirrhotics. Neurology 2004; 62:762-66

26. Sugimoto R, Iwasa M, Maeda M, et al. Value of the apparent diffusion coefficient for quantification of low-grade hepatic encephalopathy. Am J Gastroenterol 2008;103:1413-20

27. Kale RA, Gupta RK, Saraswat VA, et al. Demonstration of interstitial cerebral edema with diffusion tensor MR imaging in type $\mathrm{C}$ hepatic encephalopathy. Hepatology 2006;43:698-706

28. Nath K, Saraswat VA, Krishna YR, et al. Quantification of cerebral edema on diffusion tensor imaging in acute-on-chronic liver failure. NMR Biomed 2008;21:713-22

29. Saksena S, Rai V, Saraswat VA, et al. Cerebral diffusion tensor imaging and in vivo proton magnetic resonance spectroscopy in patients with fulminant hepatic failure. J Gastroenterol Hepatol 2008; 23:e111-19

30. Rai V, Nath K, Saraswat VA, et al. Measurement of cytotoxic and interstitial components of cerebral edema in acute hepatic failure by diffusion tensor imaging. J Magn Reson Imaging 2008;28:334-41

31. Zhang LJ, Yin JZ, Qi J, et al. Metabolic changes of anterior cingulate cortex in patients with hepatic cirrhosis: a magnetic resonance spectroscopy study. Hepatol Res 2010;40:777-85

32. Mardini H, Smith FE, Record CO, et al. Magnetic resonance quantification of water and metabolites in the brain of cirrhotics following induced hyperammonaemia. J Hepatol 2011;54:1154-60.

33. Guevara M, Baccaro ME, Gómez-Ansón M, et al. Cerebral magnetic resonance imaging reveals marked abnormalities of brain tissue density in patients with cirrhosis without overt hepatic encephalopathy. J Hepatol 2011;55:564-73

34. Lee JH, Seo DW, Lee YS, et al. Proton magnetic resonance spectroscopy (1H-MRS) findings for the brain in patients with liver cirrhosis reflect the hepatic functional reserve. Am J Gastroenterol 1999; $94: 2206-13$

35. Naegele T, Grodd W, Viebahn R, et al. MR imaging and $1 \mathrm{H}$ spectroscopy of brain metabolites in hepatic encephalopathy: time-course of renormalization after liver transplantation. Radiology 2000;216:683-91

36. Timmermnan L, Butz M, Gross J, et al. Impaired cerebral oscillatory processing in hepatic encephalopathy. Clin Neurophysiol 2007; 119:265-72

37. Gore JC. Principles and practice of functional MRI of the human brain. J Clin Invest 2003;112:4-9

38. Zafiris O, Kircheis G, Rood HA, et al. Neural mechanism underlying impaired visual judgement in the dysmetabolic brain: an fMRI study. Neuroimage 2004;22:541-52

39. Pizoli CE, Shah MN, Snyder AZ, et al. Resting-state activity in development and maintenance of normal brain function. Proc Natl Acad Sci U S A 2011;108:11638-43

40. Rosazza C, Minati L. Resting-state brain networks: literature review and clinical applications. Neurol Sci 2011;32:773-85.

41. Zhang LJ, Yang G, Yin J, et al. Abnormal default-mode network activation in cirrhotic patients: a functional magnetic resonance imaging study. Acta Radiol 2007;48:781-87

42. Zhang L, Qi R, Wu S, et al. Brain default-mode network abnormalities in hepatic encephalopathy: a resting-state functional MRI study. Hum Brain Mapp 2013:33:1384-92 